\title{
Overcoming the OTDR Dead-Zone Using a Few-Mode Fiber
}

\author{
Islam Ashry, ${ }^{\mathrm{a}, \dagger}$ Yuan Mao, ${ }^{\mathrm{a}, \dagger}$ Biwei Wang, ${ }^{\mathrm{a}, \mathrm{b}}$ Frode Hveding, ${ }^{\mathrm{c}, \#}$ Tien Khee Ng, ${ }^{\mathrm{a}}$ and Boon S. Ooi ${ }^{\mathrm{a},{ }^{*}}$ \\ ${ }^{a}$ King Abdullah University of Science and Technology (KAUST), Computer, Electrical, and \\ Mathematical Sciences and Engineering, Thuwal 23955-6900, Saudi Arabia \\ bepartment of Electronic Engineering, the Hong Kong Polytechnic University, Hong Kong SAR, \\ China \\ ${ }^{c}$ EXPEC Advanced Research Center, Saudi Aramco, Dhahran 34464, Saudi Arabia \\ ${ }^{\dagger}$ These authors contributed equally to this work \\ \#frode.hveding@aramco.com, *boon.ooi@kaust.edu.sa
}

\begin{abstract}
Optical-time-domain-reflectometer (OTDR) suffers from the existence of dead-zones along a deployed fiber under test (FUT). Within a dead-zone, OTDR typically fails to provide any reliable diagnostic information. We here use a fewmode fiber (FMF) to completely cancel the OTDR dead-zone produced by the front facet reflection of the FUT. In particular, we launch the optical pulses in the form of the $\mathrm{LP}_{01}$ mode into the FMF, and meanwhile we record the Rayleigh signal from the higher-order modes. The developed system successfully monitors the amplitude and frequency of a vibration event produced by a piezoelectric transducer (PZT) located within the dead-zone.
\end{abstract}

Keywords: Optical time-domain reflectometer, optical fiber sensors, optical fibers, optical sensors.

\section{INTRODUCTION}

Optical time-domain reflectometer (OTDR) is an important instrument used to diagnose and characterize fiber optics [1]. For example, along a fiber under test (FUT), OTDR can locate faults and measure attenuation. Besides, OTDR has been included in many distributed fiber optic sensing systems [2]. In particular, OTDR-based distributed fiber optic sensors can monitor temperature [3], vibration [4], pressure [5], among others. As a result, OTDR has been considered a crucial technology for many applications, including agricultural [6] and oil-gas industries [7].

The commercially available OTDR systems have a common drawback, the presence of dead-zones along a FUT. The dead-zones are formed because of intense Fresnel reflections produced by the fiber's front facet, connectors, splicing points, etc. Compared with the power of the backscattered Rayleigh signal used to functionalize the OTDR, the power of Fresnel reflection is much higher, which saturates the receiver and creates dead-zones. Depending on the photodetector (PD) bandwidth and the width of the OTDR's optical pulses, the dead-zones are in the range of tens to hundreds meters [4]. Within a dead-zone, unfortunately, the typical OTDR is incapable of producing reliable sensing or diagnostic information. In some critical applications, such as intrusion detection using the $\Phi$-OTDR-based fiber optic distributed acoustic sensing (DAS) [8], missing information within any section along the FUT is considered a severe problem.

Some techniques have been reported in literature to overcome the OTDR dead-zone $[9,10]$. In particular, the timecorrelated single photon-counting (TC-SPC) OTDR was introduced to cancel the dead-zones via detecting the backscattered Raman signals instead of the Rayleigh ones [9]. However, using the TC-SPC OTDR is limited to specific applications, which do not rely on Rayleigh backscattering. Alternatively, a digital signal processing (DSP) technique was reported to discard the impact of the OTDR dead-zones [10]; however, the DSP-improved OTDRs are relatively sophisticated. Another straightforward method to mitigate the effect of dead-zones is using a dummy fiber between the OTDR interrogation unit and the FUT, such that the dead-zone occurs within the dummy fiber instead of the FUT [11]. However, the added dummy fiber decreases the sensing range of the OTDR system. Additionally, at the connection point between the dummy fiber and FUT, a dead-zone might be created at the beginning of the FUT. Furthermore, the dummy fiber should have a connector/adapter that matches the FUT's connector, when joining them together. Equipping the OTDR system with dummy fibers that have all possible connector combinations would be expensive. 
Here, we report using a few-mode fiber and a photonics lantern mode-division multiplexer/demultiplexer (MUX/DEMUX) to completely cancel the dead-zone produced by the fiber's front facet. Although standard single-mode fiber (SMF) and multimode fiber (MMF) are commonly used for sensing applications, it is typically difficult to tune the characteristics of the SMF- and MMF-based sensors. In contrast, FMF has unique and flexible specifications to offer OTDR-based [12], multi-parameter [13], and absorption-based [14] sensing, among others [15]. Consequently, this work would be highly beneficial to the emerging FMF-based sensing technology.

The general operation principle of the reported FMF-based OTDR is described in Fig. 1, where a photonic lantern mode MUX/DEMUX is placed between the OTDR transceiver and the FMF. Suppose the FMF supports propagating $N$ linearly-polarized (LP) modes, the photonic lantern should have $N$ SMF ports and one FMF port. In case of injecting the OTDR optical pulses through the $\mathrm{LP}_{01}$ port, two various kinds of signals are received in the backward direction. The first signal is the Fresnel reflection, which occurs at the circulator and connector. As shown in Fig. 1, the Fresnel reflection only affects the receiver of the $\mathrm{LP}_{01}$ port. The second signal in the backward direction is the elastic Rayleigh backscattering, produced along the FMF. This work does not consider the inelastic Brillouin and Raman scattering since they are not impacted by the dead-zone. The backscattered Rayleigh signal includes all of the modes supported by the FMF [16]. Since the Fresnel reflection only affects the $\mathrm{LP}_{01}$ mode, the Rayleigh signals of the higher order modes become dead-zone free. The backscattered Rayleigh signal in the form of the $\mathrm{LP}_{11 \mathrm{a}}$ mode, for example, can diagnose the entire FMF, including the dead-zone region of the $\mathrm{LP}_{01}$ mode. This operation principle can be generalized such that any mode can be used for injecting the optical pulses, while the other modes become dead-zone free.

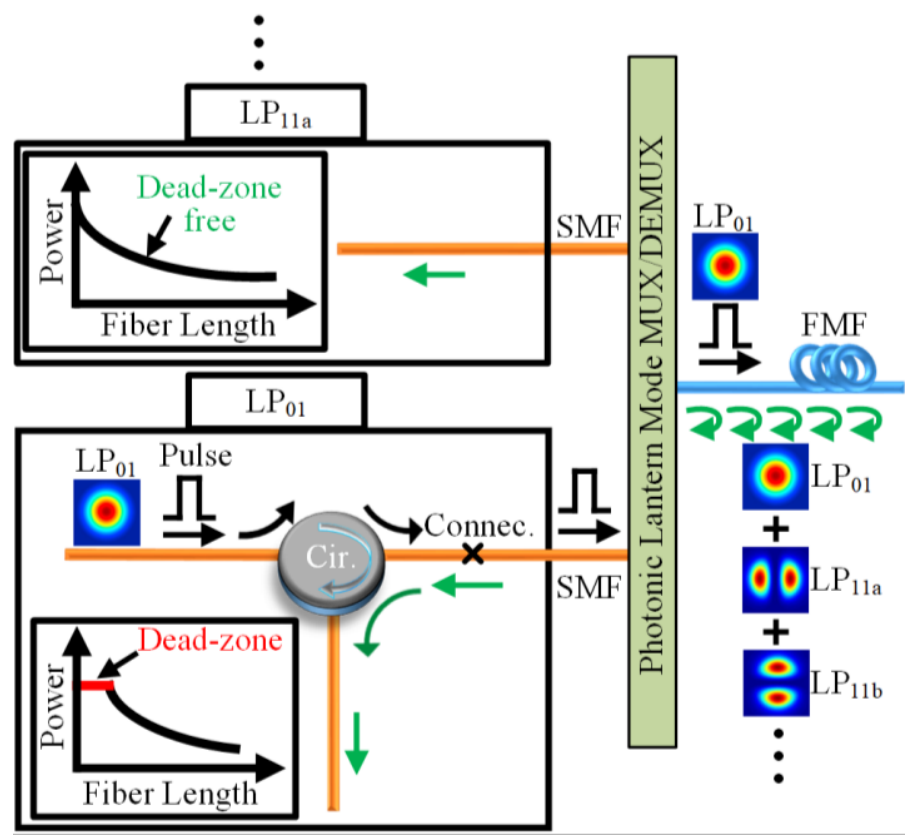

Figure 1. The introduced OTDR system, designed using the FMF and photonic lantern mode MUX/DEMUX. Connec., connector; Cir., circulator.

\section{SMF-BASED DISTRIBUTED ACOUSTIC SENSOR}

To validate the operation principle of our design, we compare the performance of two $\Phi$-OTDR DAS [4], designed using the standard SMF and a two-mode fiber (TMF). The fundamental purposes of using the fiber optic DAS are locating vibration events along the FUT and calculating the vibration frequencies [4]. Figure 2 shows the experimental design of the typical SMF-based DAS. A narrow linewidth laser produces a continuous wave (CW) light of a 1535-nm wavelength and a 40-mW optical power. The CW light is then converted into optical pulses via an acousto-optic modulator (AOM). By controlling an arbitrary waveform generator (AWG) to drive the AOM, we generate the optical pulses with a 30-ns width and $20-\mathrm{kHz}$ repetition rate. After amplification with an erbium-doped fiber amplifier (EDFA1), the optical pulses are launched through a circulator into a SMF of a $\sim 2.1-\mathrm{km}$ length. At a $\sim 2-\mathrm{m}$ distance from the input port of the SMF, 
we wind a $\sim 2-\mathrm{m}$ section of the fiber around a piezoelectric transducer (PZT1) cylinder. Meanwhile, near the distal end of the SMF, we wind a 2-m section of the fiber around another PZT cylinder (PZT2). The two PZT cylinders are identical and driven with the same function generator to produce vibrations of a $5-\mathrm{kHz}$ frequency. The backscattered Rayleigh signal from the SMF is guided via a circulator to another erbium-doped fiber amplifier (EDFA2), which amplified spontaneous emission (ASE) noise is filtered out with a fiber Bragg grating (FBG). The filtered Rayleigh signal is then detected by a photodetector and sampled with a digitizer.

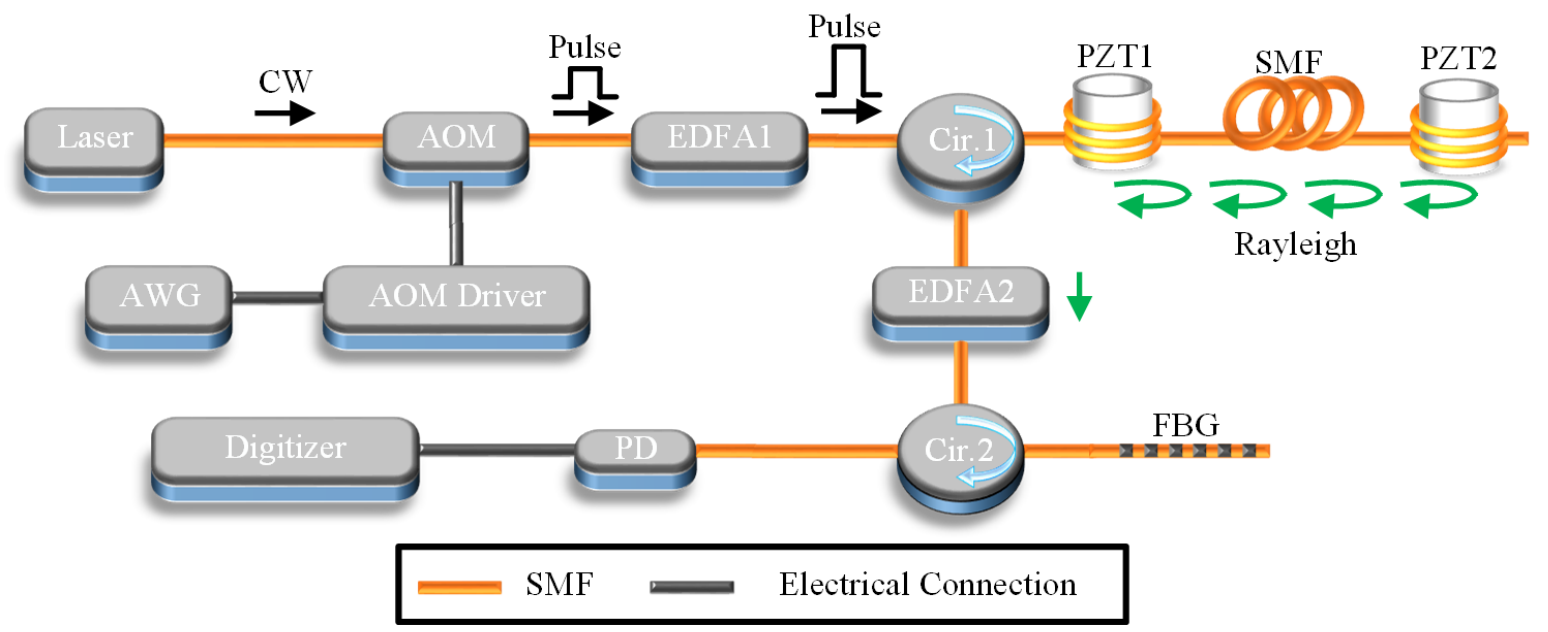

Figure 2. Experimental setup of the $\Phi$-OTDR DAS, designed using a SMF.
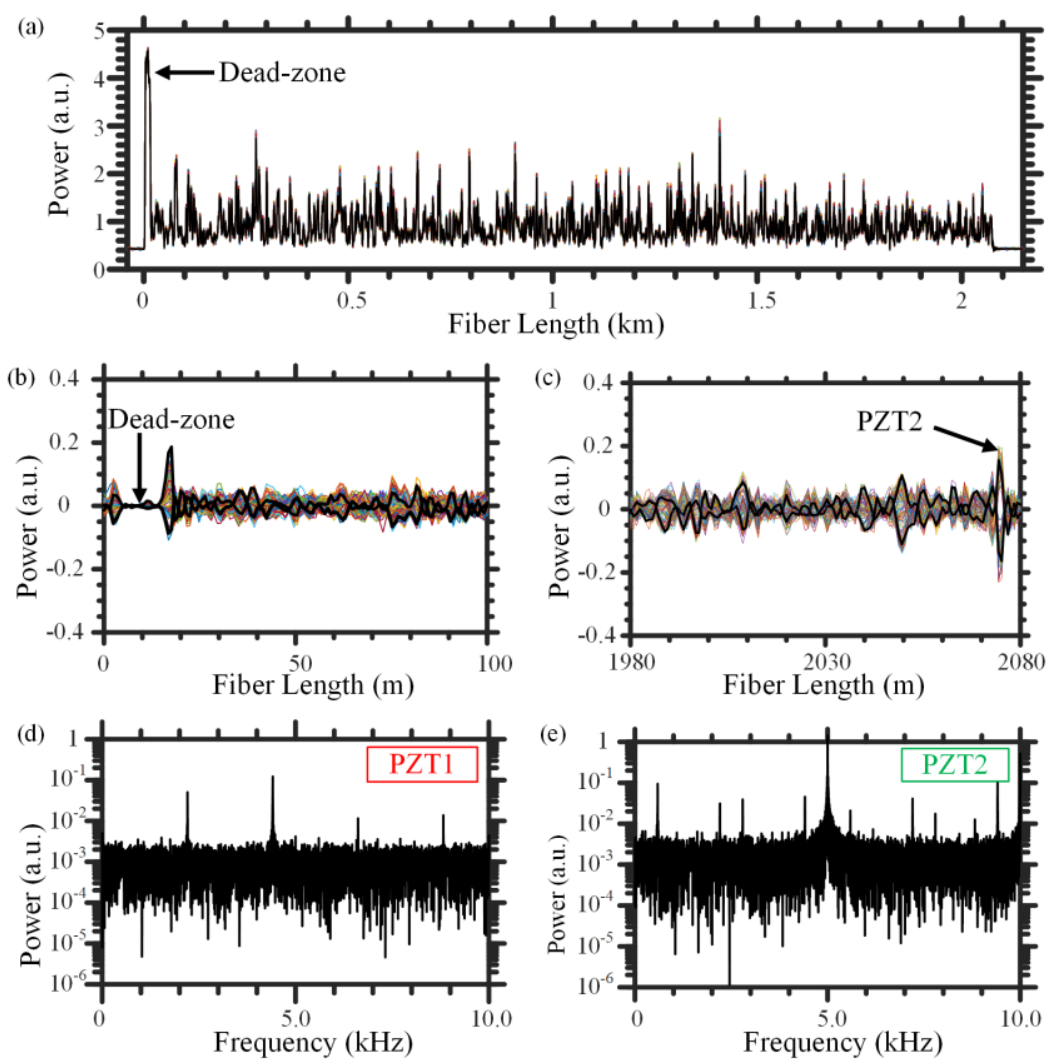

Figure 3. (a) Ten consecutive Rayleigh traces recorded along the entire SMF. Temporal vibration information of the PZT1 (b) and PZT2 (c). Power spectra of the vibration at the PZT1 (d) and PZT2 (e). 
Figure 3(a) shows 10 consecutive Rayleigh traces recorded along the entire SMF. Clearly, at the beginning of the SMF, there is a dead-zone of power that saturates the PD and larger than the typical Rayleigh signal recorded along the remaining fiber. Applying the normalized differential method [17] to the Rayleigh traces to locate the vibration events along the SMF, Figs. 3(b) and 3(c) show the vibration information of the PZT1 and PZT2, respectively. As shown in Fig. 3 (b), there is no information regarding the PZT1 vibration because it is located within the dead-zone. In other words, the outcome of the normalized differential method is roughly zeros around the PZT1's location since the PD is saturated. In contrast, the vibration information of the PZT2 is clear and can be easily distinguished from the background noise [Fig. 3(c)]. For further verification, we calculate the fast Fourier transform (FFT) of the temporal vibration signal at the PZT1 and PZT locations. The power spectrum of the signal at the PZT1 includes spectral noise [Fig. 3(d)], mainly because of the dead-zone. On the other hand, the power spectrum of the PZT2 vibration has a sharp peak at $5 \mathrm{kHz}$, the frequency produced by the function generator that drives the two PZT cylinders. The results of Fig. 3 confirm the challenges introduced by the SMF-based OTDR.

\section{FMF-BASED DISTRIBUTED ACOUSTIC SENSOR}

The other fiber optic DAS that includes our reported design is shown in Fig. 4. The setup in Fig. 4 resembles that of Fig. 2 , with two exceptions. Firstly, the used FUT is a TMF that supports propagating the $\mathrm{LP}_{01}, \mathrm{LP}_{11 \mathrm{a}}$, and $\mathrm{LP}_{11 \mathrm{~b}}$ mode at the 1535-nm operation wavelength. Secondly, we insert a 3x1 photonic lantern MUX/DEMUX between the circulator (Cir. 1) and the TMF. At a $\sim 2-\mathrm{m}$ separation from the input port of the TMF, we wind a $2-\mathrm{m}$ section of the TMF around a PZT cylinder. The vibration events produced by the PZT is located within the dead-zone, if exists. As a representative example, we launch the optical pulses of the DAS system through the $\mathrm{LP}_{01}$ port [Fig. 4].

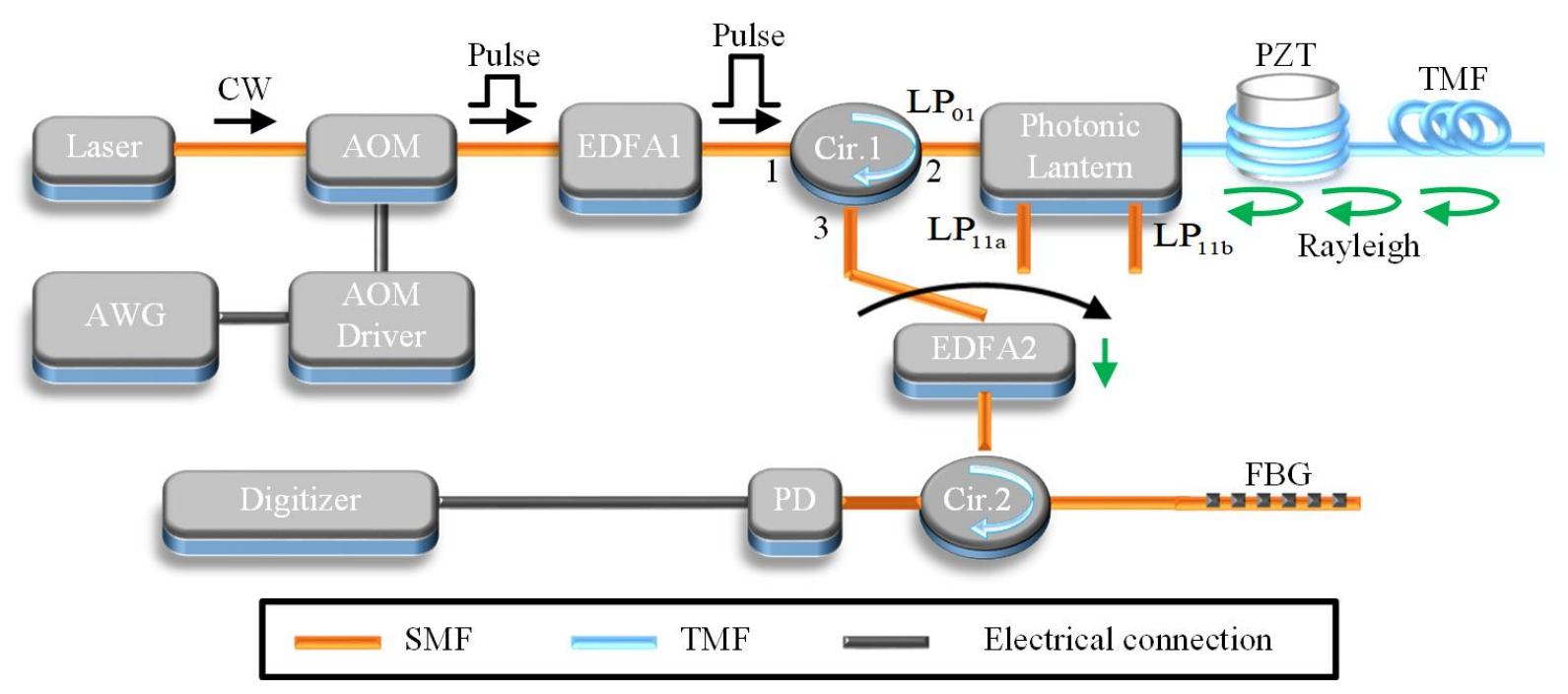

Figure 4. Experimental setup of the $\Phi$-OTDR DAS, designed using a TMF.

We initially collect the backscattered Rayleigh signal at the same $\mathrm{LP}_{01}$ port. In this scenario, the recorded Rayleigh traces still suffer from the dead-zone at the beginning of the TMF [Fig. 5(a)]. Applying the normalized differential method to the Rayleigh traces, Fig. 5(b) shows that the DAS system fails to distinguish the PZT vibration from the background noise. As explained in Fig. 1, the Rayleigh signal in the form of the $\mathrm{LP}_{01}$ mode still includes the dead-zone because the pulses are injected from its port. For further confirmation, the power spectrum of the PZT vibration includes spectral noise [Fig. 5(c)]. Alternatively, we record the Rayleigh traces at the $\mathrm{LP}_{11 \text { a }}$ mode port [Fig. 6(a)]. Clearly, the power level of the signal at the start of the TMF resembles that along the remaining fiber. In other words, the dead-zone disappears at the $\mathrm{LP}_{11 \text { a }}$ port. To validate the cancellation of the dead-zone, we apply the normalized differential method to the Rayleigh traces. As respectively shown in Figs. 6(b) and 6(c), the temporal variation and power spectrum of the PZT's vibration event are remarkable. The power spectrum has a sharp peak at $5 \mathrm{kHz}$, which matches the vibration frequency of the PZT. For further clarification, Fig. 6(d) provides a two-dimensional map to the temporal vibration around the PZT location. The $\mathrm{LP}_{11 \mathrm{a}}$ can easily detects the PZT vibration, even within the dead-zone region of the $\mathrm{LP}_{01}$ mode. 

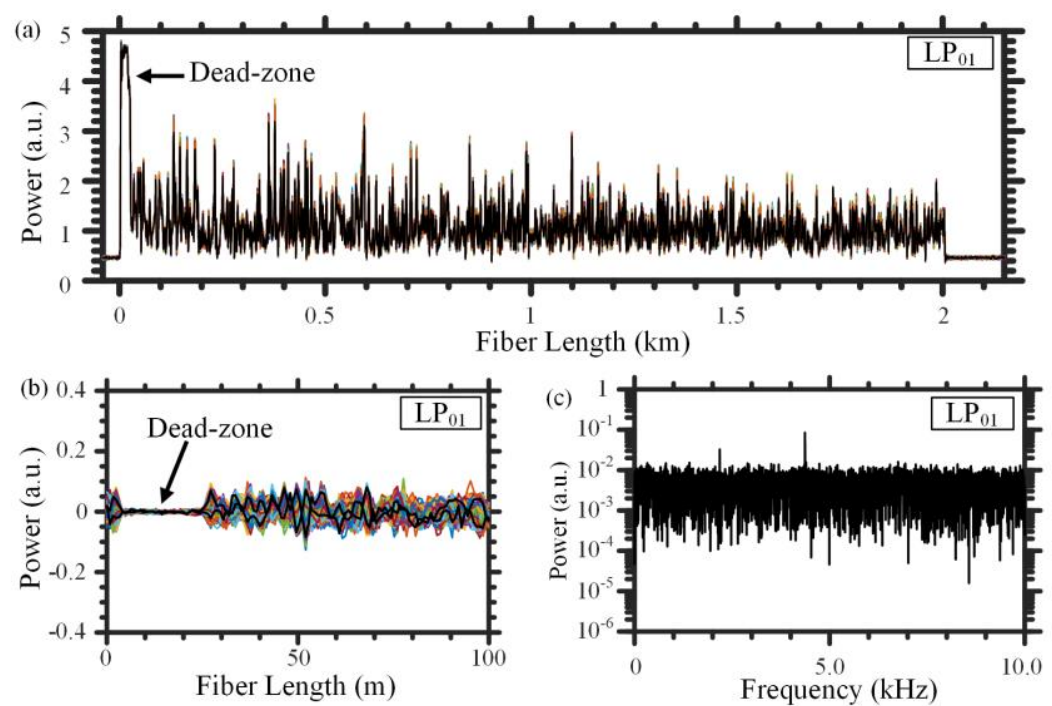

Figure 5. (a) Ten consecutive Rayleigh traces recorded along the TMF. Vibration information (b) and power spectrum (c) of the PZT cylinder located within the dead-zone of the $\mathrm{LP}_{01}$ mode.
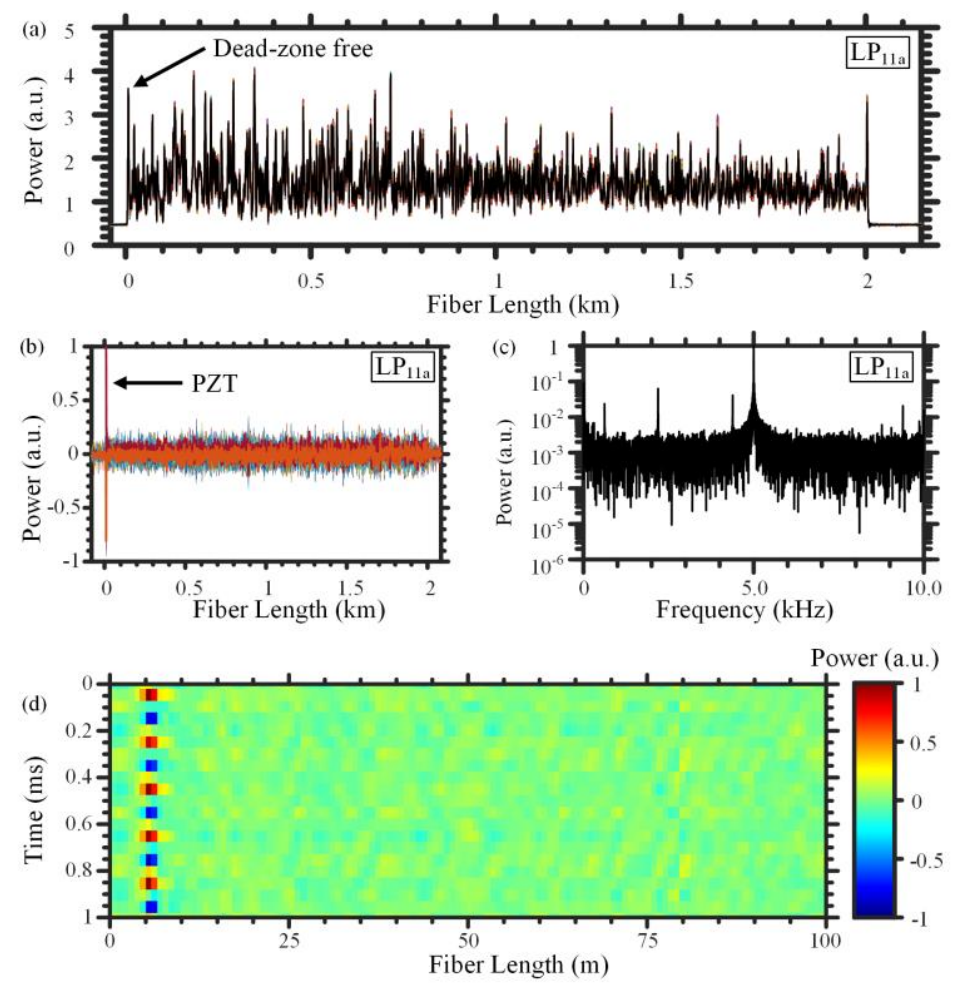

Figure 6. (a) Ten consecutive Rayleigh traces recorded along the TMF. Vibration information (b) and power spectrum (c) of the PZT cylinder, measured at the $\mathrm{LP}_{11 a}$ port. (d) Temporal evolution of the PZT vibration, when using the $\mathrm{LP}_{11 \mathrm{a}}$ Rayleigh signal.

\section{CONCLUSION}

We use a TMF and a photonic lantern mode MUX/DEMUX to overcome the dead-zone produced by the Fresnel reflection of the fiber's front facet. As a proof-of-concept demonstration, we inject the $\Phi$-OTDR-based DAS system's optical pulses as $\mathrm{LP}_{01}$ mode, while we collect the backscattered Rayleigh signal in the form of the $\mathrm{LP}_{11 \text { a }}$ mode. The 
higher order $\mathrm{LP}_{11 \mathrm{a}}$ mode's Rayleigh signal is not impacted by the Fresnel reflection and can detect the vibration even of a PZT cylinder, located within the dead-zone of the fundamental mode. Considering the evolution of using FMFs for OTDR-based sensing, the introduced design would find many potential applications.

\section{ACKNOWLEDGEMENTS}

The authors gratefully acknowledge the financial support provided to this work by the Saudi Aramco under Grant No. RGC/3/3138-01. This work is partially supported by King Abdullah University of Science and Technology (KAUST) baseline funding (BAS/1/1614-01-01), KAUST equipment funding KCR/1/2081-01-01, and GEN/1/6607-01-01.

\section{REFERENCES}

[1] Tateda, M. and Horiguchi, T., “Advances in Optical Time-Domain Reflectometry,” J. Light. Technol. 7(8), 1217-1224 (1989).

[2] Mao, Y., Ashry, I., Hveding, F., Bukhamsin, A. Y., Hong, Y., Ng, T. K. and Ooi, B. S., "Simultaneous Distributed Acoustic and Temperature Sensing Using a Multimode Fiber," IEEE J. Sel. Top. Quantum Electron. 26 (2020).

[3] Ma, X., Chang, J., Wang, Z., Wang, W. and Li, T., "Using multiple reference points in Raman based distributed temperature sensor for eliminating DC interference,” IEEE Sens. J. 14(1), 295-301 (2014).

[4] Bao, X., Zhou, D. P., Baker, C. and Chen, L., "Recent Development in the Distributed Fiber Optic Acoustic and Ultrasonic Detection," J. Light. Technol. 35(16), 3256-3267 (2017).

[5] Zhang, L., Yang, Z., Szostkiewicz, Ł., Markiewicz, K., Nasilowski, T. and Thévenaz, L., "Fully distributed pressure sensing with ultra-high-sensitivity using side-hole fibers," Opt. InfoBase Conf. Pap. Part F124-OFS (2018).

[6] Ashry, I., Mao, Y., Al-Fehaid, Y., Al-Shawaf, A., Al-Bagshi, M., Al-Brahim, S., Ng, T. K. and Ooi, B. S., "Early detection of red palm weevil using distributed optical sensor," Sci. Rep. 10(1), 3155 (2020).

[7] Yamate, T., Fujisawa, G. and Ikegami, T., "Optical Sensors for the Exploration of Oil and Gas," J. Light. Technol. 35(16), 3538-3545 (2017).

[8] Juarez, J. C. and Taylor, H. F., "Field test of a distributed fiber-optic intrusion sensor system for long perimeters," Appl. Opt. 46(11), 1968-1971 (2007).

[9] Feced, R., Farhadiroushan, M. and Handerek, V. A., "Zero dead-zone OTDR with high-spatial resolution for short haul applications,” IEEE Photonics Technol. Lett. 9(8), 1140-1142 (1997).

[10] Fernández, M. P., Bulus Rossini, L. A., Pascual, J. P. and Costanzo Caso, P. A., "DSP-enhanced OTDR for detection and estimation of events in PONs," arXiv (2018).

[11] Feuerstein, R. J., "Field measurements of deployed fiber," Opt. InfoBase Conf. Pap. (2005).

[12] Mao, Y., Ashry, I., Alias, M. S., Ng, T. K., Hveding, F., Arsalan, M. and Ooi, B. S., "Investigating the Performance of a Few-Mode Fiber for Distributed Acoustic Sensing," IEEE Photonics J. 11(5) (2019).

[13] Weng, Y., Ip, E., Pan, Z. and Wang, T., "Single-end simultaneous temperature and strain sensing techniques based on Brillouin optical time domain reflectometry in few-mode fibers," Opt. Express 23(7), 9024 (2015).

[14] Ashry, I., Wang, A. and Xu, Y., "Mode-Based Reconstruction of Chemical Distributions in Optical Fibers," IEEE J. Sel. Top. Quantum Electron. 23(2), 229-237 (2017).

[15] Ashry, I., Mao, Y., Trichili, A., Wang, B., Ng, T. K., Alouini, M.-S. and Ooi, B. S., "A Review of Using FewMode Fibers for Optical Sensing,” IEEE Access 8, 179592-179605 (2020).

[16] Wang, Z., Wu, H., Hu, X., Zhao, N., Mo, Q. and Li, G., "Rayleigh scattering in few-mode optical fibers," Sci. Rep. 6 (2016).

[17] Ashry, I., Mao, Y., Alias, M. S., Ng, T. K., Hveding, F., Arsalan, M. and Ooi, B. S., "Normalized differential method for improving the signal-to-noise ratio of a distributed acoustic sensor," Appl. Opt. 58(18), 4933 (2019). 